\title{
The use of the Nursing Activities Score in clinical settings: an integrative review
}

\author{
Como o instrumento Nursing Activities Score tem sido \\ utilizado na prática clínica: revisão integrativa \\ Cómo el instrumento Nursing Activities Score se ha utilizado \\ en la práctica clínica: revisión integradora
}

Joanie Lachance ${ }^{1}$, Frédéric Douville ${ }^{1}$, Clémence Dallaire ${ }^{1}$, Katia Grillo Padilha ${ }^{2}$, Maria Cecilia Gallani ${ }^{1}$

${ }^{1}$ Université Laval, Faculté des Sciences Infirmières, Quebec, Canada.

${ }^{2}$ Universidade de São Paulo, Escola de Enfermagem, Departamento de Enfermagem Médico-Cirúrgica, São Paulo, SP, Brazil.

\section{ABSTRACT}

Objective: analyze how studies have approached the results obtained from the application of the Nursing Activities Score (NAS) based on Donabedian's model of healthcare organization and delivery. Method: CINAHL and PubMed databases were searched for papers published between 2003 and March 2015. Results: 36 articles that met the inclusion criteria were reviewed and double-coded by three independent coders and analyzed based on the three elements of Donabedian's health care quality framework: structure, process and outcome. The most frequently addressed, but not always tested, variables were those that fell into the structure category. Conclusion: variables that fell into the process category were used less frequently. Beside NAS, the most frequently used variables in the outcome category were mortality and length of stay. However, no study used a quality framework for healthcare or NAS to evaluate costs, and it is recommended that further research should explore this approach.

\section{DESCRIPTORS}

Workload; Nursing Staff; Intensive Care Units; Quality of Health Care; Review. 


\section{INTRODUCTION}

Intensive care units (ICUs) are constantly evolving and in recent decades have had to deal with an increasing number of old patients and comorbidities. This in turn leads to a greater number of complex procedures ${ }^{(1)}$, which calls for closer monitoring and a greater need for nursing interventions, resulting in increased nurse workload and higher costs.

In fact, nursing resources are the largest single component of direct ICU costs, accounting for nearly $50 \%$ of the ICU budget ${ }^{(2)}$. Thus, the use of more objective strategies to evaluate nursing activities is essential to optimize the use of ICU resources and ensure appropriateness, efficacy and cost-effectiveness ${ }^{(3)}$. Besides this, in the current global context, marked by resource constraints and nursing shortages, it is imperative to portray a more accurate and comprehensive picture of nursing and the value and benefits of the services they provide in line with preestablished objectives and standards ${ }^{(4)}$. The use of tools for nursing workload measurement serves as a starting point for the analysis, comparison, and processing of data related to nursing activities and can provide important inputs to promote the optimization of the use of resources and establish a more adequate nurse-patient ratio $^{(3)}$.

Many attempts have been made to characterize nursing activities in $\operatorname{ICUs}_{s}^{(3)}$. Beginning with the Therapeutic Intervention Scoring System (TISS) $)^{(5)}$, a number of different tools have been developed, such as the Italian Time-oriented Score System (TOSS), the Canadian Project Research in Nursing (PRN), the TISS-28(6) and the Nine Equivalents of Nursing Manpower use score (NEMS) ${ }^{(7)}$; the latter two of which evaluate severity of illness and complexity of therapeutic procedures. However, it is known that the relationship between severity of illness and the time spent on nursing interventions is not linear, since severity accounts for only part of the variability of nursing activities. Therefore, in 2003, the Nursing Activities Score (NAS) was developed to provide an overall description of all nursing activities in ICUs, not only those correlated to the severity of illness ${ }^{(2)}$. It has been shown that NAS represents about $80 \%$ of the work activities of nurses in ICUs, which is substantially more representative that the $43 \%$ obtained using TISS-28(2). NAS has been used in over a dozen countries ${ }^{(8)}$ and a number of papers have been published regarding its clinical application. Some studies have demonstrated a relationship between NAS and outcomes such as mortality and adverse events; however, this association is not constant throughout all studies and this inconstancy is yet to be explained.

Nursing care performance involves "the analysis of multiple interacting elements that relate to the diverse aspects of nursing services, their antecedents and their results", reflecting "nursing care as a complex, aggregate entity, comprised of multiple interrelated and interdependent subsystems and components that are logically coordinated and oriented toward the achievement of common goals"(4). In this sense, Donabedian's model of healthcare organiza- tion and delivery guides understanding on how inputs are acquired from the (nursing) care environment and fed into the service production cycle, where transformation of resources results in changes in patient conditions ${ }^{(9)}$.

In light of the above, this integrative review assesses how studies have approached the results obtained using this tool based on Donabedian's model of healthcare organization and delivery and aims to contextualize the use of NAS across the healthcare continuum to gain further insight into the interpretation of its results.

\section{METHOD}

The review included the following steps: problem identification, literature search, data extraction, and data analysis and reporting ${ }^{(10)}$. The PICO strategy was used to formulate the question, whereby the population was defined as "Intensive Care Units" and intervention as "application of the Nursing Activities Score - NAS”. Comparison criteria were not applied and outcome was analyzed from an exploratory perspective, in accordance with Donabedian's model ${ }^{(9)}$.

Problem identification: the central question of this integrative review was: "what are the main purposes for using NAS in ICU settings? The following aspects were analyzed: study objective, the context in which NAS was used, sample size (for patient and NAS application), the strategy used for applying the tool, and the purpose of using NAS, according to the SPO elements of Donabedian's model: structure/process/outcome.

Search strategy: a search was conducted of the CINAHL and PubMed databases of articles produced between 2003, which was when the first article on NAS was produced, and March 2015, using the following concepts: 1) "Nursing Activities Score" or "Nursing Activity Score" and "scale"; 2) "intensive care units" or "critical care"; 3) "workload measurement" or "workload". This search strategy was adapted to the terminology of the databases. In addition, the bibliographies of potentially eligible studies were analyzed manually to identify studies not brought up by the search strategy.

Eligibility: the following inclusion criteria were used - 1) fully peer-reviewed papers published in English, French, Italian, Portuguese or Spanish; 2) papers should demonstrate the use of NAS in a clinical setting; and 3) papers should indicate the context in which the tool was used (for example type of ICU - adult, pediatric, general, clinical); 4) papers should specify the method of NAS application; and 5) specify the purpose for using the tool. Both exploratory and correlational studies were included since they met the mentioned criteria. Studies addressing cross-cultural adaptation, conceptual aspects of the tool, and the measurement properties of NAS were excluded; as were published abstracts or papers without abstracts found to be lacking information on the context in which the NAS was used, methods of application, or the purpose for using the tool during data extraction. Articles reporting the use of NAS in settings other than ICUs were excluded, considering that the original focus of the tool were $\mathrm{ICUs}_{\mathrm{s}}{ }^{(2)}$. 
The studies were sorted by title and abstract by JL in order to select the articles that met the inclusion criteria. Duplicated articles were discarded. Thereafter, the full text of the articles that met the inclusion criteria were inde- pendently screened by JL, FD and MCG and compared. A total of 36 papers were selected. Details of the methodological steps are shown in a flowchart developed in accordance with the PRISMA Statement ${ }^{(11)}$ (Figure 1).
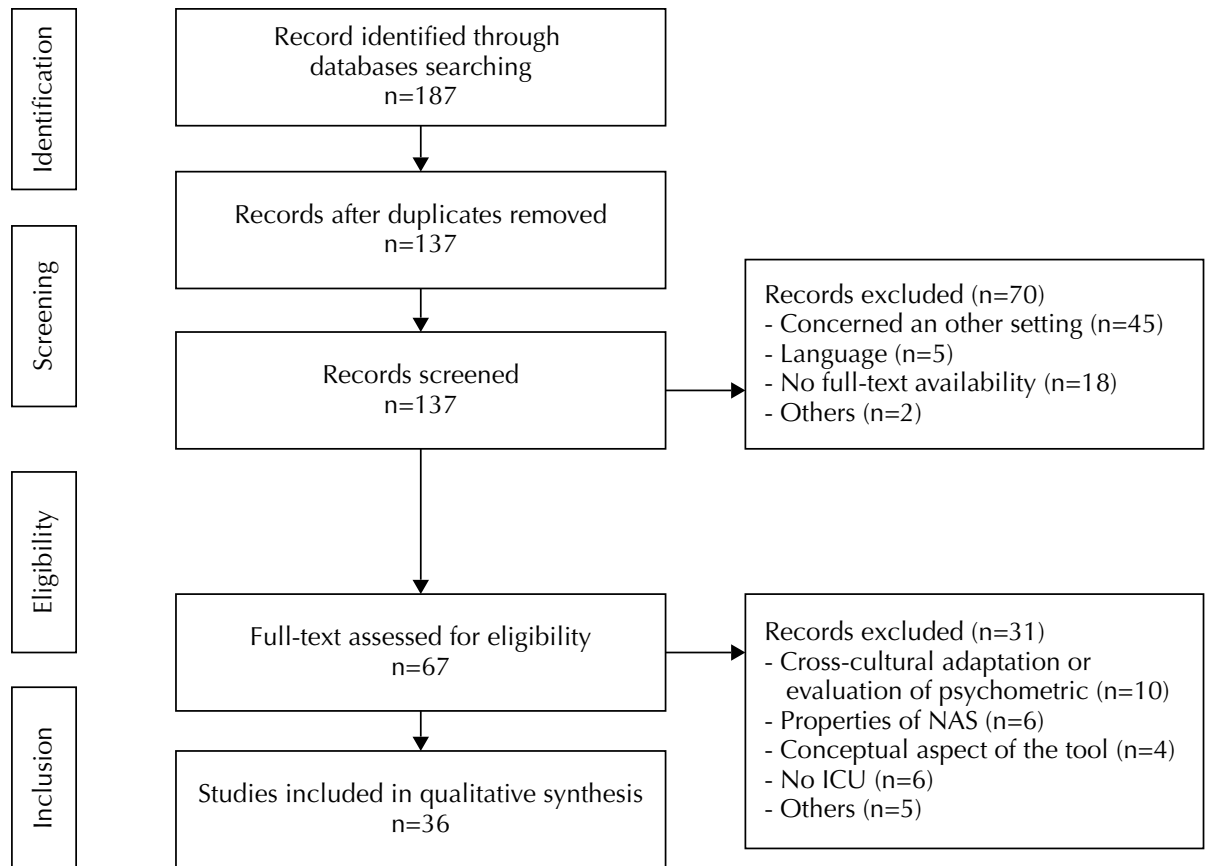

Figure 1 - Flowchart showing the methodological steps of the integrative review.

Data extraction: first coding was carried out with papers to check agreement of data extraction and to confirm the quality of the coding sheet. Where there was disagreement between the two reviewers, the final decision was taken after discussion with reviewer MCG for language reasons.

Data analysis: NAS serves to assess the percentage of time spent on nursing interventions delivered to an ICU patient. Thus, the results obtained with NAS could serve as one of the means to evaluate the quality of health services provided by nurses in ICU. A multitude of models or frameworks were developed for health services quality improvements such as Donabedian ${ }^{(9)}$ and more recently ${ }^{(4)}$. Since Donabedian framework is already largely used in nursing studies to evaluate quality of care ${ }^{(12-13)}$, this model was chosen to analyze the retained studies.

Donabedian's framework evaluates three elements of the quality of health service delivery: structure, process and outcome, whereby each component is interdependent and influenced by the other components ${ }^{(9)}$. Structure refers to all technical, human and organizational resources related to the service or institution, and stable patient characteristics, such as age and gender. In this study, other patient characteristics at the time of admission that affect process and cannot be influenced by the nurse were included in the structure category, such as clinical severity, patient origin, and reason for admission to ICU. Process includes all activities carried out by professionals involved in direct patient care, care coordination, and the interaction between and response to these activities. Outcome includes changes in the health status of a patient after direct contact with the health system such as healing, survival, mortality and adverse events. It should be noted that this analysis of the SPO does not imply a separation between means and ends, but rather an unbroken care process chain continuum ${ }^{(9)}$. Thus, NAS can be seen as a connecting element between the structure and process components, since it enables the quantification of the nursing activities that characterize the process.

\section{RESULTS}

A large majority of the articles reviewed by this study were published in nursing journals (86.1\%), while 9.3\% were published in multidisciplinary journals ${ }^{(14-49)}$ (Chart 1). With regard to the nursing journals, $24 \%$ were journals specifically focusing on critical care or cardiac nursing, while the rest were general nursing journals. Year of publication varied from 2005 to 2014, with a major concentration (81.4\%) in the period 2010 to 2014, indicating that the use of NAS for clinical purposes is a more recent phenomenon. Most articles were produced in Brazil (69.4\%), followed by Europe (27.8\%) and one publication (2.8\%) from China. Patient sample size varied from 34 to 5,856; only one paper failed to mention the number of patients who participated in the study. Sixteen articles did not clearly state the number of NAS applications. Those articles that stated the number of applications reported between 34 and 28,390 applications including patient follow-up. The large amount of variability between the samples made it impossible to compare study data. All studies indicated the context in which NAS was used, the application method, and the purpose for using the tool. 
Chart 1 - Summary of the studies of the application of NAS in clinical settings analyzed by the present study.

\begin{tabular}{|c|c|c|c|c|c|c|c|}
\hline \multirow{2}{*}{$\begin{array}{l}\text { Author, year/country } \\
\text { Journal }\end{array}$} & \multirow{2}{*}{ Aim } & \multirow{2}{*}{$\begin{array}{c}\text { Sample } \\
\text { (application } \\
\text { of NAS) }\end{array}$} & \multirow{2}{*}{$\begin{array}{c}\text { Context } \\
\text { (type of ICU) }\end{array}$} & \multirow{2}{*}{$\begin{array}{l}\text { Strategy used for } \\
\text { applying the tool }\end{array}$} & \multicolumn{3}{|c|}{ Evaluating the Quality of Care (Donabedian, 1978) } \\
\hline & & & & & Structure & Process & Outcome \\
\hline $\begin{array}{l}\text { Adell et al. }{ }^{(14)} \text {, } \\
\text { 2005/Spain Enferm } \\
\text { Intensiva. }\end{array}$ & $\begin{array}{l}\text { To evaluate NAS } \\
\text { results throughout the } \\
\text { hospitalization. }\end{array}$ & $\begin{array}{l}\mathrm{N}_{\text {patient }}=250 \\
\mathrm{~N}_{\mathrm{NAS}}=1,880\end{array}$ & General adult ICU & $\begin{array}{c}3 \text { times/day (per } \\
\text { shift) } \\
\text { and then average } \\
\text { NAS/day }\end{array}$ & $\begin{array}{l}\text {-Age -Gender } \\
\text {-Special clinical } \\
\text { conditions } \\
\text {-Origin* }\end{array}$ & -NAS & $\begin{array}{l}\text {-Mortality* } \\
\text {-LOS }\end{array}$ \\
\hline $\begin{array}{l}\text { Altafin et al. }{ }^{(15)}, 2014 \text { / } \\
\text { Brazil } \\
\text { Rev Bras Ter } \\
\text { Intensiva. }\end{array}$ & $\begin{array}{l}\text { To evaluate nursing } \\
\text { workload. }\end{array}$ & $\begin{array}{l}\mathrm{N}_{\text {Patient }}=437 \\
\mathrm{~N}_{\mathrm{NAS}}=\mathrm{NS}\end{array}$ & Adult ICU & $\begin{array}{c}\text { Every } 24 \mathrm{~h} \text { at } \\
\text { 7a.m. }\end{array}$ & $\begin{array}{l}\text {-Age* -Gender* } \\
\text {-Reason for admission* } \\
\text {-Origin* -Severity index } \\
\text { (APACHE II)* -Organ } \\
\text { Failure (SOFA)* }\end{array}$ & $\begin{array}{l}\text {-NAS } \\
\text {-TISS-28* }\end{array}$ & $\begin{array}{c}\text {-LOS* } \\
\text {-Mortality* }\end{array}$ \\
\hline $\begin{array}{l}\text { Argibay-Lago et al. }{ }^{(16)} \text {, } \\
\text { 2014/Spain } \\
\text { Enferm Clin. }\end{array}$ & $\begin{array}{l}\text { To assess nursing } \\
\text { workload among } \\
\text { nurses providing } \\
\text { special treatment to } \\
\text { patients. }\end{array}$ & $\begin{array}{l}N_{\text {patient }}=46 \\
N_{\text {NAS }}=N S\end{array}$ & $\begin{array}{l}>1 \text { ICU patients } \\
\text { after suffering } \\
\text { sudden cardiac } \\
\text { arrest, with } \\
\text { or without } \\
\text { therapeutic } \\
\text { hypothermia }\end{array}$ & Every $24 \mathrm{~h}$ & $\begin{array}{l}\text {-Age -Gender } \\
\text {-Clinical data }\end{array}$ & $\begin{array}{l}\text {-NAS } \\
\text {-TISS } 28 \\
\text {-NEMS }\end{array}$ & $\begin{array}{l}\text {-Mortality* } \\
\text {-Neurological } \\
\text { evolution* }\end{array}$ \\
\hline $\begin{array}{l}\text { Camuci et al. }{ }^{(17)} \text {, } \\
\text { 2014/Brazil } \\
\text { Rev Latino Am } \\
\text { Enfermagem. }\end{array}$ & $\begin{array}{l}\text { To evaluate nursing } \\
\text { workload in a burns } \\
\text { intensive care unit. }\end{array}$ & $\begin{array}{c}N_{\text {patient }}=50 \\
N_{\text {NAS }}=1,221\end{array}$ & Burns ICU & Every $24 \mathrm{~h}$ & $\begin{array}{l}\text {-Age } \\
\text {-Gender } \\
\text {-Burn data }\end{array}$ & -NAS & \\
\hline $\begin{array}{l}\text { Carmona-Monge et } \\
\text { al. }{ }^{(18)}, 2013 / \text { Spain } \\
\text { Rev Esc Enferm USP. }\end{array}$ & $\begin{array}{c}\text { To compare the NAS } \\
\text { in two ICUs. }\end{array}$ & $\begin{array}{l}N_{\text {patient }}=103 \\
N_{\text {NAS }}=941\end{array}$ & $\begin{array}{l}\text { Two Clinical adult } \\
\text { ICUs }\end{array}$ & Every $24 \mathrm{~h}$ & $\begin{array}{c}\text {-Age } \\
\text {-Gender } \\
\text {-Reason for admission } \\
-2 \text { types of ICU }\end{array}$ & -NAS & $\begin{array}{l}\text {-LOS } \\
\text {-Mortality }\end{array}$ \\
\hline $\begin{array}{l}\text { Carmona-Monge et } \\
\text { al. }{ }^{(19)}, 2013 / \text { Spain } \\
\text { Rev Esc Enferm USP. }\end{array}$ & $\begin{array}{c}\text { To assess nursing } \\
\text { workload from } \\
\text { admission to discharge } \\
\text { among } 3 \text { groups of } \\
\text { ICU patients. }\end{array}$ & $\begin{array}{l}\mathrm{N}_{\text {patient }}=563 \\
\mathrm{~N}_{\mathrm{NAS}}=5,704\end{array}$ & Medical ICU & Every $24 \mathrm{~h}$ & $\begin{array}{c}\text {-Age } \\
\text {-Gender } \\
\text {-Reason for admission* } \\
\text {-Ratio } 1: 2,1: 3\end{array}$ & $\begin{array}{c}\text {-NAS } \\
\text {-Invasive procedure }\end{array}$ & $\begin{array}{l}\text {-LOS } \\
\text {-Mortality }\end{array}$ \\
\hline $\begin{array}{l}\text { Ciampone et al. }{ }^{(20)}, \\
\text { 2006/Brazil } \\
\text { Acta Paul Enferm. }\end{array}$ & $\begin{array}{l}\text { To compare nursing } \\
\text { care needs of elderly } \\
\text { and non-elderly } \\
\text { patients. }\end{array}$ & $\begin{array}{l}\mathrm{N}_{\text {patient }}=50 \\
\mathrm{~N}_{\mathrm{NAS}}=\mathrm{NS}\end{array}$ & Adult ICU & Every $24 \mathrm{~h}$ & $\begin{array}{l}\text {-Age* }- \text { Gender } \\
\text {-Comorbidities }\end{array}$ & $\begin{array}{c}\text {-NAS } \\
\text {-Type of treatment }\end{array}$ & $\begin{array}{c}\text {-LOS } \\
\text {-Destination } \\
\text {-Mortality }\end{array}$ \\
\hline $\begin{array}{l}\text { Coelho et al. }{ }^{(21)} \text {, } \\
\text { 2011/Brazil } \\
\text { Texto Contexto } \\
\text { Enferm. }\end{array}$ & $\begin{array}{l}\text { To identify nursing } \\
\text { workload and its } \\
\text { association with } \\
\text { demographic and } \\
\text { clinical patient's data. }\end{array}$ & $\begin{array}{l}\mathrm{N}_{\text {patient }}=100 \\
\mathrm{~N}_{\mathrm{NAS}}=100\end{array}$ & $\begin{array}{l}\text { Cardiac Intensive } \\
\text { Care Unit }\end{array}$ & $\begin{array}{l}\text { Once, } 24 \mathrm{~h} \text { after } \\
\text { admission }\end{array}$ & $\begin{array}{c}\text { - Age* -Gender* } \\
\text { Origin* -Reason for } \\
\text { admission* } \\
\text {-Organic dysfunction } \\
\text { (LODS)* -Severity index } \\
\text { (SAPS II)* -Mortality risk* }\end{array}$ & -NAS & $\begin{array}{c}\text {-LOS* } \\
\text {-Mortality* }\end{array}$ \\
\hline $\begin{array}{l}\text { Cremasco et al }{ }^{(22)} \text {, } \\
\text { 2013/Brazil } \\
\text { J Clin Nurs. }\end{array}$ & $\begin{array}{l}\text { To verify the } \\
\text { association between } \\
\text { nursing workload and } \\
\text { pressure ulcer risk and } \\
\text { illness severity. }\end{array}$ & $\begin{array}{l}\mathrm{N}_{\text {patient }}=160 \\
N_{\text {NAS }}=N S\end{array}$ & University ICU & Every $24 \mathrm{~h}$ & $\begin{array}{l}\text {-Age -Gender } \\
\text {-Severity index (SAPS II)* } \\
\text {-Pressure ulcer risk } \\
\text { (Braden Scale)* } \\
\text {-Reason for readmission }\end{array}$ & -NAS & $\begin{array}{c}\text {-PU } \\
\text { Development* } \\
\text {-LOS* }\end{array}$ \\
\hline $\begin{array}{l}\text { Cyrino et al.(23), } \\
\text { 2012/Brazil } \\
\text { Esc Anna Nery. }\end{array}$ & $\begin{array}{l}\text { To analyze the } \\
\text { impact of the Patient } \\
\text { Classification System } \\
\text { on the care process and } \\
\text { nosocomial infection. }\end{array}$ & $\begin{array}{c}\mathrm{N}_{\text {patient }}=214 \\
\mathrm{~N}_{\mathrm{NAS}}=\mathrm{NS}\end{array}$ & $\begin{array}{c}\text { Adult } \\
\text { Medical-Surgical } \\
\text { ICU }\end{array}$ & Every $24 \mathrm{~h}$ & $\begin{array}{c}\text {-Age* -Gender } \\
\text {-Reason for readmission } \\
\text {-Patient classification } \\
\text { System* } \\
\text {-Bed occupancy rate }\end{array}$ & -NAS & $\begin{array}{l}\text {-Nosocomial } \\
\text { infection* } \\
\text {-Mortality* } \\
\text {-LOS* }\end{array}$ \\
\hline $\begin{array}{l}\text { Silva et al. }{ }^{(24)}, \\
\text { 2010/Brazil } \\
\text { Rev Latino Am } \\
\text { Enfermagem. }\end{array}$ & $\begin{array}{l}\text { To identify factors } \\
\text { related to discharge } \\
\text { of ICU patients } \\
\text { in hospitals with } \\
\text { intermediate care } \\
\text { units. }\end{array}$ & $\begin{array}{c}\mathrm{N}_{\text {patient }}=600 \\
\mathrm{~N}_{\mathrm{NAS}}=\mathrm{NS}\end{array}$ & 4 General ICUs & $\begin{array}{l}\text { Twice Every } 24 \mathrm{~h} \\
\text { After admission } \\
\text { to discharge }\end{array}$ & $\begin{array}{c}\text {-Age -Gender } \\
\text {-Comorbidities } \\
\text {-Severity index (SAPS II) } \\
\text {-Beds ratio ICU : IU -Risk } \\
\text { of death (SAPS II) } \\
\text {-Organ dysfunction }\end{array}$ & -NAS & $\begin{array}{l}\text {-Mortality * } \\
\text {-LOS }\end{array}$ \\
\hline $\begin{array}{l}\text { Silva et al. }{ }^{(25)} \text {, } \\
\text { 2011/Brazil } \\
\text { Rev Latino Am } \\
\text { Enfermagem. }\end{array}$ & $\begin{array}{l}\text { To identify factors } \\
\text { associated with death } \\
\text { and readmission. }\end{array}$ & $\begin{array}{l}N_{\text {patient }}=600 \\
N_{\text {NAS }}=1,200\end{array}$ & $\begin{array}{l}2 \text { General public } \\
\text { and } 2 \text { private ICUs }\end{array}$ & $\begin{array}{l}\text { Twice } 24 \mathrm{~h} \\
\text { Admission/ } \\
\text { discharge }\end{array}$ & $\begin{array}{c}\text {-Age -Gender } \\
\text {-Origin -Mortality } \\
\text { risk (SPAS II) -Organ } \\
\text { dysfunction (LODS) }\end{array}$ & -NAS & $\begin{array}{l}\text {-Mortality * } \\
\text { Readmission* } \\
\text {-LOS }\end{array}$ \\
\hline $\begin{array}{l}\text { Daud-Gallotti et al. } \\
\text { (26), 2012/Brazil } \\
\text { PLOS ONE. }\end{array}$ & $\begin{array}{c}\text { To evaluate the } \\
\text { association between } \\
\text { nursing workload and } \\
\text { HAl. }\end{array}$ & $\begin{array}{l}\mathrm{N}_{\text {patient }}=195 \\
\mathrm{~N}_{\mathrm{NAS}}=\mathrm{NS}\end{array}$ & $\begin{array}{l}3 \text { Medical ICUs } \\
\text { and } 1 \text { step-down } \\
\text { beds unit }\end{array}$ & $\begin{array}{l}\text { Every } 24 \mathrm{~h} \\
\text { Every shift ( } 3 \\
\text { times) }\end{array}$ & $\begin{array}{c}\text {-Age -Gender } \\
\text {-Origin -Severity index } \\
\text { (APACHE II) and SAPS } \\
\text { II) -Organ dysfunction } \\
\text { (SOFA) } \\
\text {-Comorbidities } \\
\text {-Nurse-patient ratio } \\
\text {-Absenteeism }\end{array}$ & $\begin{array}{c}\text {-NAS } \\
\text {-compliance with } \\
\text { the nurses' care plan } \\
\text {-Procedures } \\
\text {-Communication } \\
\text { failure }\end{array}$ & $\begin{array}{l}\text {-HAI* } \\
\text {-LOS } \\
\text {-Adverse } \\
\text { events }\end{array}$ \\
\hline
\end{tabular}




\begin{tabular}{|c|c|c|c|c|c|c|c|}
\hline \multirow{2}{*}{$\begin{array}{l}\text { Author, year/country } \\
\text { Journal }\end{array}$} & \multirow{2}{*}{ Aim } & \multirow{2}{*}{$\begin{array}{l}\text { Sample } \\
\text { (application } \\
\text { of NAS) }\end{array}$} & \multirow{2}{*}{$\begin{array}{c}\text { Context } \\
\text { (type of ICU) }\end{array}$} & \multirow{2}{*}{$\begin{array}{l}\text { Strategy used for } \\
\text { applying the tool }\end{array}$} & \multicolumn{3}{|c|}{ Evaluating the Quality of Care (Donabedian, 1978) } \\
\hline & & & & & Structure & Process & Outcome \\
\hline $\begin{array}{l}\text { Sousa et al. }{ }^{(27)}, \\
\text { 2008/Brazil } \\
\text { Rev Latino Am } \\
\text { Enfermagem. }\end{array}$ & $\begin{array}{l}\text { To compare illness } \\
\text { severity and nursing } \\
\text { workload and to } \\
\text { identify predictors } \\
\text { of nursing workload } \\
\text { for the treatment of } \\
\text { elderly patients. }\end{array}$ & $\begin{array}{l}\mathrm{N}_{\text {patient }}=71 \\
\mathrm{~N}_{\mathrm{NAS}}=71\end{array}$ & General & $\begin{array}{l}\text { Every } 24 \mathrm{~h} \\
\text { at } 8 \text { a.m. }\end{array}$ & $\begin{array}{c}\text {-Age* -Gender } \\
\text {-Severity index (SAPS II) }\end{array}$ & -NAS & -Mortality \\
\hline $\begin{array}{l}\text { Feitosa et al. }{ }^{(28)}, \\
\text { 2012/Brazil } \\
\text { Esc Anna Nery. }\end{array}$ & $\begin{array}{c}\text { To evaluate the } \\
\text { demand for nursing } \\
\text { care. }\end{array}$ & $\begin{array}{l}\mathrm{N}_{\text {patient }}=45 \\
\mathrm{~N}_{\mathrm{NAS}}=328\end{array}$ & $\begin{array}{l}\text { Two General Adult } \\
\text { Clinical-Surgical } \\
\text { ICUs }\end{array}$ & Every $24 \mathrm{~h}$ & $\begin{array}{l}\text {-Age } \\
\text {-Gender }\end{array}$ & $\begin{array}{l}\text {-NAS } \\
\text {-Type of treatment } \\
\text { (related to diagnosis) }\end{array}$ & $\begin{array}{c}\text {-LOS } \\
\text {-Mortality }\end{array}$ \\
\hline $\begin{array}{l}\text { Giakoumidakis et } \\
\text { al. }^{(29)}, 2011 / \text { Greece } \\
\text { Nurs Crit Care. }\end{array}$ & $\begin{array}{l}\text { To identify the factors } \\
\text { affecting the length of } \\
\text { stay in hospital. }\end{array}$ & $\begin{array}{l}N_{\text {patien }} \mathrm{t}=313 \\
\mathrm{~N}_{\text {NAS }}=313\end{array}$ & $\begin{array}{c}\text { Cardiac surgery } \\
\text { ICU }\end{array}$ & $\begin{array}{l}\text { Every } 24 \mathrm{~h} \\
\text { Once }\end{array}$ & $\begin{array}{l}\text {-Age -Gender } \\
\text {-Comorbidities } \\
\text {-Perioperative } \\
\text { and mortality risk } \\
\text { (EuroSCORE) }\end{array}$ & $\begin{array}{l}\text {-NAS } \\
\text {-Type of surgery } \\
\text {-Procedure }\end{array}$ & -LOS* \\
\hline $\begin{array}{l}\text { Gerasimou-Angelidi } \\
\text { et al. }{ }^{(30)}, 2014 / \text { Greece } \\
\text { J Nurs Manag. }\end{array}$ & $\begin{array}{l}\text { To study the } \\
\text { association between } \\
\text { family satisfaction and } \\
\text { nursing workload. }\end{array}$ & $\begin{array}{c}\mathrm{N}_{\text {patient }}=106 \\
\mathrm{~N}_{\mathrm{NAS}}=\mathrm{NS}\end{array}$ & Adult ICU & $\begin{array}{l}\text { Three times/day } \\
\text { (per shift) }\end{array}$ & $\begin{array}{c}\text {-Age, } \\
\text {-Severity index (SAPS II) } \\
\text { (covariables) }\end{array}$ & -NAS & $\begin{array}{l}\text {-Family } \\
\text { Satisfaction } \\
\text { ICU } 24 \text { (FS } \\
\text { ICU-24)* } \\
\text {-LOS* }\end{array}$ \\
\hline $\begin{array}{l}\text { Giakoumidakis et } \\
\text { al. }{ }^{(31)}, 2012 / \text { Greece } \\
\text { Eur J Cardiovasc } \\
\text { Nurs. }\end{array}$ & $\begin{array}{l}\text { To identify the risk } \\
\text { factors for increased } \\
\text { in-hospital mortality. }\end{array}$ & $\begin{array}{l}N_{\text {Patient }}=313 \\
N_{\text {NAS }}=313\end{array}$ & Cardiac surgery & $\begin{array}{l}\text { Every } 24 \mathrm{~h} \\
\text { Once at } \\
\text { admission }\end{array}$ & $\begin{array}{c}\text {-Age -Gender } \\
\text {-Comorbidities } \\
\text {-BMl -Surgery and } \\
\text { Mortality risk (EuroScore) }\end{array}$ & $\begin{array}{c}\text {-NAS } \\
\text {-Type of surgery }\end{array}$ & $\begin{array}{l}\text {-Mortality* } \\
\text {-LOS }\end{array}$ \\
\hline $\begin{array}{l}\text { Gonçalves et al. }{ }^{(32)} \text {, } \\
\text { 2006/Brazil } \\
\text { Rev Bras Enferm. }\end{array}$ & $\begin{array}{l}\text { To determine the } \\
\text { daily nursing care } \\
\text { needs. }\end{array}$ & $\begin{array}{l}\mathrm{N}_{\text {patient }}=50 \\
\mathrm{~N}_{\mathrm{NAS}}=\mathrm{NS}\end{array}$ & Adult ICU & Every $24 \mathrm{~h}$ & $\begin{array}{c}\text {-Age* -Gender } \\
\text {-Reason for admission } \\
\text {-Origin* }\end{array}$ & $\begin{array}{c}\text {-NAS } \\
\text {-Type of treatment }\end{array}$ & $\begin{array}{l}\text {-Mortality* } \\
\text {-LOS* }\end{array}$ \\
\hline $\begin{array}{l}\text { Gonçalves et al. }{ }^{(33)} \text {, } \\
\text { 2007/Brazil } \\
\text { Rev Esc Enferm USP. }\end{array}$ & $\begin{array}{l}\text { To analyze nursing } \\
\text { workload on the first } \\
\text { day of admission and } \\
\text { associated factors. }\end{array}$ & $\begin{array}{l}N_{\text {patient }}=214 \\
N_{N A S}=214\end{array}$ & $\begin{array}{l}5 \text { Adult private } \\
\text { ICUs -3 } \\
\text { general and } 2 \\
\text { neurological }\end{array}$ & $\begin{array}{l}\text { Once, } 24 \mathrm{~h} \text { after } \\
\text { admission }\end{array}$ & $\begin{array}{l}\text {-Age -Gender } \\
\text {-Diagnosis }\end{array}$ & $\begin{array}{c}\text {-NAS } \\
\text {-Type of treatment }\end{array}$ & $\begin{array}{c}\text {-Mortality* } \\
\text {-LOS* }\end{array}$ \\
\hline $\begin{array}{l}\text { Inoue et al. }{ }^{(34)}, 2010 / \\
\text { Brazil } \\
\text { Acta Paul Enferm. }\end{array}$ & $\begin{array}{l}\text { To analyze nurse- } \\
\text { patient ratios based } \\
\text { on NAS. }\end{array}$ & $\begin{array}{l}N_{\text {patient }}=107 \\
N_{\text {NAS }}=N S\end{array}$ & & Every $24 \mathrm{~h}$ & $\begin{array}{l}\text {-Age -Gender } \\
\text {-Reason for admission } \\
\text {-Nurse-patient ratio* }\end{array}$ & $\begin{array}{c}\text {-NAS } \\
\text {-Type of treatment }\end{array}$ & $\begin{array}{c}\text {-Destination } \\
\text {-LOS }\end{array}$ \\
\hline $\begin{array}{l}\text { Kakushi et al. } .^{(35)} \text {, } \\
\text { 2014/Brazil } \\
\text { Rev Latino Am } \\
\text { Enfermagem. }\end{array}$ & $\begin{array}{l}\text { To identify direct and } \\
\text { indirect nursing-care } \\
\text { time. }\end{array}$ & $\begin{array}{c}N_{\text {patient }}=N S \\
N_{\text {NAS }}=24,886\end{array}$ & $\begin{array}{l}\text { Children and adult } \\
\text { private ICU }\end{array}$ & Every 24 h, & $\begin{array}{c}\text {-Age } \\
\text {-Reason for admission } \\
\text {-Categories of nursing } \\
\text { team } \\
\text {-Bed occupancy rate }\end{array}$ & $\begin{array}{l}\text {-NAS (direct care) } \\
\text {-Indirect care* }\end{array}$ & -LOS \\
\hline $\begin{array}{l}\text { Lago et al. }{ }^{(36)}, 2011 / \\
\text { Brazil } \\
\text { Int J Palliat Nurs. }\end{array}$ & $\begin{array}{l}\text { To evaluate the } \\
\text { provision of medical } \\
\text { and nursing care to } \\
\text { children in the last } 24 \\
\text { hours of life }\end{array}$ & $\begin{array}{l}N_{\text {patient }}=34 \\
N_{N A S}=34\end{array}$ & Pediatric ICU & $\begin{array}{c}\text { Every } 24 \mathrm{~h} \\
\text { Once in the last } \\
24 \mathrm{~h} \text { of life before } \\
\text { death }\end{array}$ & $\begin{array}{c}\text {-Age, } \\
\text {-Gender } \\
\text {-Ratio 1: 1-2 }\end{array}$ & $\begin{array}{c}\text {-NAS } \\
\text {-Decision making } \\
\text {-Procedure } \\
\text {-Type of treatment* } \\
\text { (RCR or not RCR) }\end{array}$ & $-\mathrm{LOS}$ \\
\hline $\begin{array}{l}\text { Lucchini et al. }{ }^{(37)} \text {, } \\
2011 \text { /Italy } \\
\text { Assist Inferm Ric. }\end{array}$ & $\begin{array}{c}\text { To assess the } \\
\text { association between } \\
\text { adverse events, nurse } \\
\text { staffing levels and } \\
\text { workload. }\end{array}$ & $\begin{array}{c}\mathrm{N}_{\text {patient }}=240 \\
N_{N A S}=N S\end{array}$ & $\begin{array}{l}\text { Adult Clinical and } \\
\text { Surgical ICU }\end{array}$ & Every $24 \mathrm{~h}$ & $\begin{array}{l}\text {-Age } \\
\text {-Ratio nurse-patient* } \\
\text {-Bed occupancy rate* }\end{array}$ & NAS & $\begin{array}{l}\text {-Adverse } \\
\text { events* }\end{array}$ \\
\hline $\begin{array}{l}\text { Lucchini et } \text { al. }^{38)} \text {, } \\
2012 \text { /Italy } \\
\text { Assist Inferm Ric. }\end{array}$ & $\begin{array}{l}\text { To detect the impact } \\
\text { of ventilation mode } \\
\text { on nursing workload. }\end{array}$ & $\begin{array}{l}N_{\text {patient }}=200 \\
N_{N A S}=N S\end{array}$ & General adult ICU & Every $24 \mathrm{~h}$ & & $\begin{array}{c}\text {-Ventilation mode* } \\
\text {-NAS }\end{array}$ & \\
\hline $\begin{array}{l}\text { Lucchini et al. }{ }^{(39)} \\
2014 / \text { Italy } \\
\text { Intensive Crit Care } \\
\text { Nurs. }\end{array}$ & $\begin{array}{l}\text { To analyze the } \\
\text { application of the } \\
\text { Nursing Activities } \\
\text { Score in an intensive } \\
\text { care department. }\end{array}$ & $\begin{array}{l}\mathrm{N}_{\text {patient }}=5,856 \\
N_{\text {NAS }}=28,390\end{array}$ & $\begin{array}{c}\text { General, } \\
\text { Neurologic and } \\
\text { Cardiothoracic } \\
\text { Surgery ICU } \\
\text { (adult and } \\
\text { pediatric) }\end{array}$ & $\begin{array}{l}\text { Every } 24 \mathrm{~h} \text { in } \\
\text { the morning } \\
\text { (retrospectively) }\end{array}$ & $\begin{array}{c}\text {-Age* }^{*} \\
\text {-Severity index (SAPS II } \\
\text { and SAPS III)* } \\
\text {-Pressure ulcer risk* } \\
\text { (Braden scale) }\end{array}$ & $\begin{array}{l}\text {-NAS } \\
\text {-Level of sedation } \\
\text { (RASS)* }\end{array}$ & -LOS* \\
\hline $\begin{array}{l}\text { Nogueira et al. } .^{(40)} \text {, } \\
\text { 2007/Brazil } \\
\text { Rev Bras Ter } \\
\text { Intensiva. }\end{array}$ & $\begin{array}{l}\text { To explore the } \\
\text { correlations between } \\
\text { NAS, severity index } \\
\text { and mortality. }\end{array}$ & $\begin{array}{l}\mathrm{N}_{\text {patient }}=148 \\
\mathrm{~N}_{\mathrm{NAS}}=\mathrm{NS}\end{array}$ & Adult ICU & Every $24 \mathrm{~h}$ & $\begin{array}{c}\text {-Age } \\
\text {-Gender } \\
\text {-Reason for admission } \\
\text {-Severity index } \\
\text { (APACHE II)* }\end{array}$ & -NAS & $\begin{array}{l}\text {-Mortality* } \\
\text {-LOS }\end{array}$ \\
\hline $\begin{array}{l}\text { Nogueira et al. } .^{(41)} \text {, } \\
2013 \backslash \text { Brazil } \\
\text { Rev Bras Ter } \\
\text { intensiva. }\end{array}$ & $\begin{array}{l}\text { To compare nursing } \\
\text { workloads in public } \\
\text { and private ICUs }\end{array}$ & $\begin{array}{l}N_{\text {Patient }}=600 \\
N_{\mathrm{NAS}}=1,200\end{array}$ & $\begin{array}{l}2 \text { Public and } 2 \\
\text { Private ICUs }\end{array}$ & $\begin{array}{c}\text { Every } 24 \mathrm{~h} \text { Twice } \\
\text { Admission and } \\
\text { discharge }\end{array}$ & $\begin{array}{c}\text {-Age -Gender } \\
\text {-Organ dysfunction (LODS) } \\
\text {-Severity index (SAPS II) } \\
\text {-Type of ICU* }\end{array}$ & -NAS & $\begin{array}{c}\text {-LOS } \\
\text {-Mortality* }\end{array}$ \\
\hline
\end{tabular}




\begin{tabular}{|c|c|c|c|c|c|c|c|}
\hline \multirow{2}{*}{$\begin{array}{l}\text { Author, year/country } \\
\text { Journal }\end{array}$} & \multirow{2}{*}{ Aim } & \multirow{2}{*}{$\begin{array}{c}\text { Sample } \\
\text { (application } \\
\text { of NAS) }\end{array}$} & \multirow{2}{*}{$\begin{array}{c}\text { Context } \\
\text { (type of ICU) }\end{array}$} & \multirow{2}{*}{$\begin{array}{l}\text { Strategy used for } \\
\text { applying the tool }\end{array}$} & \multicolumn{3}{|c|}{ Evaluating the Quality of Care (Donabedian, 1978) } \\
\hline & & & & & Structure & Process & Outcome \\
\hline $\begin{array}{l}\text { Nogueira et al. } .^{(42)}, \\
\text { 2014/Brazil } \\
\text { PLOS ONE. }\end{array}$ & $\begin{array}{l}\text { To identify the factors } \\
\text { related to the high } \\
\text { nursing workload } \\
\text { demanded in the } \\
\text { treatment of trauma } \\
\text { victims. }\end{array}$ & $\begin{array}{l}N_{\text {patient }}=200 \\
N_{\text {NAS }}=200\end{array}$ & Trauma ICU & After the first $24 \mathrm{~h}$ & $\begin{array}{c}\text {-Age* -Gender* } \\
\text {-AIS* -Organ } \\
\text { dysfunction(LODS)* } \\
\text {-ISS* -Origin* -Reason } \\
\text { for admission* } \\
\text {-Comorbidities* } \\
\text {-Mortality risk* (SAPS II, } \\
\text { APACHE II) }\end{array}$ & $\begin{array}{c}\text {-NAS } \\
\text {-Interval between } \\
\text { ER and ICU* }\end{array}$ & \\
\hline $\begin{array}{l}\text { Novaretti et al. } .^{(43)} \text {, } \\
2014 / \text { Brazil } \\
\text { Rev Bras Enferm. }\end{array}$ & $\begin{array}{l}\text { To identify the } \\
\text { influence of } \\
\text { nursing workload } \\
\text { on the occurrence } \\
\text { of noninjurious } \\
\text { incidents and adverse } \\
\text { events. }\end{array}$ & $\begin{array}{l}\mathrm{N}_{\text {patient }}=399 \\
\mathrm{~N}_{\mathrm{NAS}}=\mathrm{NS}\end{array}$ & $\begin{array}{l}2 \text { Clinical-Surgical } \\
\text { ICU. Patients } \square 15 \\
\text { years old }\end{array}$ & $\begin{array}{l}\text { Three times/day } \\
\text { (per shift) } \\
\text { and then, } \\
\text { average NAS/day }\end{array}$ & $\begin{array}{l}\text {-Severity index } \\
\text { (APACHE II) }\end{array}$ & -NAS & $\begin{array}{l}\text {-Mortality } \\
\text {-LOS } \\
\text {-Incidents } \\
\text { without } \\
\text { injury* } \\
\text {-Adverse } \\
\text { events* }\end{array}$ \\
\hline $\begin{array}{l}\text { Nunes et al. }{ }^{(44)}, 2013 / \\
\text { Brazil } \\
\text { Rev Latino Am } \\
\text { Enfermagem. }\end{array}$ & $\begin{array}{l}\text { To analyze nursing } \\
\text { workload related to } \\
\text { the ideal and current } \\
\text { nurse-patient ratio. }\end{array}$ & $\begin{array}{l}N_{\text {patient }}=144 \\
N_{N A S}=406\end{array}$ & Neonatal ICU & Every $24 \mathrm{~h}$ & $\begin{array}{c}\text {-Age } \\
\text {-Weight } \\
\text {-Type of unit* }\end{array}$ & -NAS & \\
\hline $\begin{array}{l}\text { Padilha et al. }{ }^{(45)} \text {, } \\
\text { 2008/Brazil } \\
\text { Intensive Crit Care } \\
\text { Nurs. }\end{array}$ & $\begin{array}{l}\text { To describe nursing } \\
\text { workload and its } \\
\text { association with } \\
\text { patient variables. }\end{array}$ & $\begin{array}{l}N_{\text {patient }}=200 \\
N_{\text {NAS }}=200\end{array}$ & $\begin{array}{l}2 \text { General and } 2 \\
\text { Neurological ICUs }\end{array}$ & $\begin{array}{l}\text { Every } 24 \mathrm{~h} \\
\text { First } 24 \mathrm{~h} \text { after } \\
\text { admission }\end{array}$ & $\begin{array}{l}\text {-Age -Gender } \\
\text {-Severity index } \\
\text { (SAPS II)* }\end{array}$ & $\begin{array}{c}\text {-NAS } \\
\text {-Therapeutic } \\
\text { interventions } \\
\text { (TISS-28)* }\end{array}$ & $\begin{array}{c}\text {-LOS* } \\
\text {-Mortality* }\end{array}$ \\
\hline $\begin{array}{l}\text { Padilha et al. }{ }^{(46)}, \\
\text { 2010/Brazil } \\
\text { Int Crit Care Nurs. }\end{array}$ & $\begin{array}{c}\text { To identify and } \\
\text { analyze daily } \\
\text { nursing workload } \\
\text { and the adequacy of } \\
\text { nursing staff. }\end{array}$ & $\begin{array}{l}\mathrm{N}_{\text {patient }}=68 \\
\mathrm{~N}_{\mathrm{NAS}}=690\end{array}$ & $\begin{array}{c}\text { Adult ICU Private } \\
\text { hospital }\end{array}$ & $\begin{array}{l}\text { Every } 6 \mathrm{~h} \text { shift, } \\
\text { Daily }\end{array}$ & $\begin{array}{c}\text {-Age -Gender } \\
\text {-Origin } \\
\text {-Occupancy Rate } \\
\text {-Nurse-patient ratio* }\end{array}$ & -NAS & $-\operatorname{LOS}$ \\
\hline $\begin{array}{l}\text { Panunto et al. }{ }^{(47)} \\
\text { 2012/Brazil } \\
\text { Acta Paul Enferm. }\end{array}$ & $\begin{array}{c}\text { To evaluate nursing } \\
\text { workload }\end{array}$ & $\begin{array}{l}\mathrm{N}_{\text {patient }}=107 \\
\mathrm{~N}_{\mathrm{NAS}}=574\end{array}$ & Adult ICU & Every $24 \mathrm{~h}$ & $\begin{array}{c}\text {-Age -Gender } \\
\text {-Reason for admission } \\
\text {-Origin }\end{array}$ & $\begin{array}{c}\text {-NAS } \\
\text {-Type of treatment }\end{array}$ & $-\operatorname{LOS}$ \\
\hline $\begin{array}{l}\text { Peng et al. }{ }^{(48)}, 2014 / \\
\text { China } \\
\text { Nurs Health Sci. }\end{array}$ & $\begin{array}{l}\text { To explore the } \\
\text { correlation between } \\
\text { patient illness } \\
\text { severity and nursing } \\
\text { workload. }\end{array}$ & $\begin{array}{l}\mathrm{N}_{\text {patient }}=229 \\
\mathrm{~N}_{\mathrm{NAS}}=\mathrm{NS}\end{array}$ & $\begin{array}{l}\text { Rescue room of } \\
\text { the Emergency } \\
\text { Department } \\
\text { (trauma) }\end{array}$ & Every $24 \mathrm{~h}$ & $\begin{array}{c}\text {-Gender } \\
\text {-Severity index } \\
\text { (APACHE- II)* } \\
\text {-Nurse-patient ratio } \\
\text {-Reason for admission }\end{array}$ & -NAS & \\
\hline $\begin{array}{l}\text { Queijo et al. }{ }^{(49)} \text {, } \\
\text { 2013/Brazil } \\
\text { Int Crit Care Nurs. }\end{array}$ & $\begin{array}{l}\text { To assess nursing } \\
\text { workload in neuro } \\
\text { ICUs and identify } \\
\text { independent factors } \\
\text { associated with } \\
\text { nursing workload }\end{array}$ & $\begin{array}{l}\mathrm{N}_{\text {patient }}=100 \\
\mathrm{~N}_{\mathrm{NAS}}=\mathrm{NS}\end{array}$ & $\begin{array}{c}\text { General, } \\
\text { Cardiovascular } \\
\text { and neurological } \\
\text { ICU }\end{array}$ & $\begin{array}{l}\text { The first } 24 \mathrm{~h} \\
\text { after admission }\end{array}$ & $\begin{array}{c}\text {-Age* -Gender* } \\
\text {-Clinical history } \\
\text {-Reason for admission* } \\
\text {-Severity index } \\
\text { (SAPS II) -Organ } \\
\text { Dysfunction (LODS) } \\
\text {-Origin* }\end{array}$ & -NAS & $\begin{array}{c}\text {-LOS } \\
\text {-Mortality* }\end{array}$ \\
\hline
\end{tabular}

*Variables tested against NAS; AIS: Abbreviated Injury Scale; APACHE: Acute Physiology and Chronic Health Evaluation; ER: Emergency Room; HAI: Healthcare-Associated Infections; ICU: Intensive Care Unit; ISS: Injury Severity Score; IU: semi-intensive unit; LODS: Logistic Organ Dysfunction System; LOS: Length of Stay; NAS: Nursing Activities Score; NEMS: Nine Equivalents of Nursing Manpower Use Score; NS: Not Specified; PU: Pressure Ulcer; SAPS: Simplified Acute Physiology Score; SOFA: Sepsis Related Organ Failure Assessment;TISS-28: Therapeutic Intervention Scoring System; RASS: Richmond Agitation Sedation Scale.

NAS was used in one or more ICUs and some studies compared different ICUs. The studies targeted different types of ICUs, and therefore patients: clinical, clinical-surgical, and specialized ICUs (cardiac, neurological, and burns). Five studies reported the use of NAS with pediatric or neonatal patients.

\section{THE USE OF NAS AND THE NURSING CARE SYSTEM}

The use of NAS across the unbroken care process chain continuum was analyzed $^{(9)}$. The variables described in the studies were categorized into one of the three elements of the framework: structure, process and outcome.

The variables that fell into the structure category were far more frequent and were related to the stable patient characteristics (age and gender), clinical profile at admission (reason for admission, origin, associated clinical conditions, organ dysfunction, illness severity and mortality risk, ulcer pressure risk) and environmental conditions (type of unit, nurse-patient ratio, bed-occupancy rate, professional background of the nursing team, absenteeism). However, it is important to highlight that these variables were not always tested against NAS: for example, age and gender were mentioned by 33 and 29 studies, respectively, but only nine studies tested the association between NAS and age and only four tested the association between NAS and gender. Severity of illness and risk of mortality were tested against NAS in 14 and four studies respectively, and only mentioned in six and two studies, respectively. Other variables tested against NAS included: clinical condition (one study), origin (five studies), reason for admission (five studies), different types of unit or distribution (four studies), risk of pressure ulcer, and bed occupancy rate (one study).

With regard to process, besides NAS, few variables were described, and even fewer variables were tested 
against NAS: TISS-28 in two studies, type of treatment (described in six studies and tested in one study), and the interval between emergency department-ICU.

With respect to outcome category, the most frequently used variables were mortality and length of stay (LOS). Mortality was mentioned in 20 papers and tested against NAS in 14 studies. LOS was measured in 28 papers, and effectively tested in 10 studies. Additionally, a range of different variables that fall into this category were used and tested against NAS by separate studies: clinical evolution, nosocomial infection, readmission, HAI, adverse events, noninjurious incidents and family satisfaction.

Finally, no study reported using a quality framework for healthcare or NAS to evaluate costs.

\section{DISCUSSION}

This literature review summarizes studies assessing the use of NAS in given contexts, application methods, and the purpose for using this tool in clinical settings.

The large majority of studies concerning the clinical use of NAS were published in nursing journals, which is to be expected given that NAS deals specifically with nursing interventions. However, some studies were published in multidisciplinary journals, which may be viewed as a strategic effort to increase the visibility of the unique role of nursing in ICUs.

The majority of studies were conducted in Brazil during the last four years, indicating that this country has contributed most to the clinical application of this tool. Considering that NAS is widely used around the world, particularly in Europe, it is very important to stimulate the application of this tool from a research perspective and the dissemination of results in Europe.

Important insights about nursing activities in ICU and their contribution to the continuum of healthcare could be gained by comparing the results of the application of NAS in different cultures. International collaborations involving multicenter studies could be an interesting way of making this possible.

NAS was applied in various types of ICUs, including neonatal and pediatric units. However, it is important to mention that NAS was developed for the adult ICU context ${ }^{(2)}$ and, to date, validation studies of the NAS in these settings do not exist.

Regarding the SPO elements of Donabedian's framework, it was observed that the most frequently described, but not always tested, variables were those related to stable patient characteristics (age and gender) and severity of illness, measured using different tools (APACHE, SAPS). A wide range of variables that fall into the structure category were also mentioned; however, the lack of a minimum set of standardized variables makes it impossible to compare the different contexts in which NAS was applied. Considering the significant impact of structure on process (and thus on nursing activities) and, consequently, on outcomes, the determination of a minimum set of variables could make a particularly important contribution to improving the analysis and interpretation of NAS results. Moreover, it is important to explore how these variables may influence NAS and outcomes.

Beside NAS, the studies mentioned few variables that fall into the process category. Type of treatment was mentioned in seven studies and tested against NAS in only one. It is important to determine which other variables in the process category influence NAS results and their effects on outcome. For example, coordination of care is a variable that potentially falls into the process category, since it is influenced by the setting and other structure variables and has a presumed causal effect on patient outcome ${ }^{(50)}$.

The most frequently measured variables in the outcome category were LOS and mortality. These are key-variables in the evaluation of ICU outcomes, since these units have higher mortality rates than other hospital units. In the United States, there are approximately four million ICU admissions per year, with average mortality rates of between 8 and 19\%, which is equivalent to around 500,000 deaths per year ${ }^{(51)}$. Furthermore, ICU is a costly component of the health care budget and this cost is largely accounted for by LOS. Despite the importance of these outcome variables, they should not be used in isolation from other variables to indicate the impact of nursing care since they reflect the contribution (or failure) of several systems of care and healthcare disciplines. LOS and mortality are part of a group of outcomes in the nursing care performance framework ${ }^{(4)}$ that reflect the joint contribution of nursing care, health status, readmission, and complications. Other quality indicators that are more sensitive to nursing interventions include: (a) patient safety (for example patient falls, injuries, medication errors, pulmonary, intravenous and urinary tract infections, pressure ulcers); (b) patient comfort and quality of life in the care context (meeting patients' needs in relation to personal hygiene, nutrition, management of symptoms such as pain and dyspnoea, and continence, and avoiding unnecessary interventions during hospitalization, such as physical or chemical restraints, nasogastric tubes or prolonged use of urinary catheters); (c) changes in patients or families' knowledge, skills, and behaviors; (d) patients' functional status (physical, psychosocial and cognitive); and (e) patient and family satisfaction with the care experience. The systematic inclusion of such variables by studies using NAS could make a significant contribution to the evaluation of the impact of nursing activities ${ }^{(4)}$.

This review also highlights the importance of a framework to guide the use of NAS in clinical practice, the elaboration of good hypotheses for studies of NAS and its implications for healthcare quality, and the interpretation of results. Furthermore, despite the fact that one of the underlying aims of NAS is to promote effective cost management and utilization of nursing resources ${ }^{(30)}$, which can account for up to $50 \%$ of the total spending of an $\mathrm{ICU}^{(2)}$, this review shows that there is a lack research exploring the use of NAS to evaluate and manage costs and further research in this area is therefore highly recommended ${ }^{(2)}$.

The present integrative review has some limitations. Considering the absence of randomized control trials, this review did not take a systematic approach or carry out a systematic comparison of study groups. Furthermore, a 
meta-analysis was not conducted due to the variability and lack of information in some studies.

\section{CONCLUSION}

This review shows that NAS has been applied in clinical settings in various types of ICUs. The analysis of the results based on Donabedian's model indicates that NAS was used to test several variables that fall into the structure category (principally age, sex and severity of illness), but few variables related to process. With regard to outcome, the most frequently tested variables were mortality and LOS, which are not nurse-sensitive variables. Further research should consider the use of a conceptual model to guide the use of NAS and the interpretation of results, and address the notion of health economics.

\section{RESUMO}

Objetivo: Analisar como os estudos têm abordado os resultados obtidos com a aplicação do Nursing Acivities Score (NAS) na perspectiva de organização do cuidado em saúde, de acordo com o modelo de Donabedian. Método: A pesquisa bibliográfica foi realizada a partir das bases de dados CINAHL e PubMed, no período compreendido entre 2003 e março de 2015 . Os 36 artigos incluídos foram revisados e codificados duplamente por três avaliadores independentes e analisados à luz dos três elementos do modelo de qualidade de cuidados de saúde: Estrutura, Processo e Resultado. Resultados: As variáveis relacionadas à Estrutura foram as mais frequentes, mas nem sempre foram testadas em relação ao NAS. Além do NAS variáveis relativas ao Processo foram menos utilizadas. No que concerne à etapa Resultado, as variáveis mortalidade e tempo de hospitalização foram as mais comuns. Conclusão: Nenhum estudo testou a hipóteses sob a perspectiva de um modelo de cuidados de saúde, assim como de custos, uma abordagem que deve ser explorada em estudos posteriores.

\section{DESCRITORES}

Carga de Trabalho; Recursos Humanos de Enfermagem; Unidades de Terapia Intensiva; Qualidade da Assistência à Saúde; Revisão.

\section{RESUMEN}

Objetivo: Analizar cómo los estudios se han ocupado de los resultados obtenidos con la aplicación del Nursing Acivities Score (NAS) en la perspectiva de organización de cuidados en salud, de acuerdo con el modelo de Donabedian. Método: Una búsqueda bibliográfica se realizó a partir de las bases de datos CINAHL y PubMed para el período entre 2003 y marzo de 2015. Resultados: El 36 artículos retenidos fueron revisados y codificados en dos ocasiones por tres evaluadores independientes y analizados a la luz de los tres elementos del modelo de calidad cuidado de la salud de Donabedian: Estructura, Proceso y Resultado. Conclusión: Las variables relacionadas con la Estructura fueron el más común, pero no siempre se probaron para el NAS. Adémas del NAS, las variables relacionadas con el Proceso fueron menos recurrentes. En cuanto a la etapa de Resultado, las variables mortalidad y duración de la estancia hospitalaria fueron los más comunes. Sin embargo, ningún estudio probó hipótesis desde la perspectiva de un modelo de organisation de la atención de salud, así como de costos, un enfoque que debe ser explorado en estudios futuros.

\section{DESCRIPTORES}

Carga de Trabajo; Personal de Enfermería; Unidades de Cuidados Intensivos; Calidad de la Atención de Salud; Revisión.

\section{REFERENCES}

1. Sprung CL, Artigas A, Kesecioglu J, Pezzi A, Wiis J, Pirracchio R, et al. The Eldicus prospective, observational study of triage decision making in European intensive care units. Part II: intensive care benefit for the elderly. Crit Care Med. 2012;40(1):132-8.

2. Miranda DR, Nap R, de Rijk A, Schaufeli W, lapichino G, System TWGTIS. Nursing Activities Score. Crit Care Med. 2003;31(2):374-82.

3. Guccione A, Morena A, Pezzi A, lapichino G. The assessment of nursing workload. Minerva Anestesiol. 2004;70(5):411-6.

4. Dubois CA, D'Amour D, Pomey MP, Girard F, Brault I. Conceptualizing performance of nursing care as a prerequisite for better measurement: a systematic and interpretive review. BMC Nurs. 2013;12:7.

5. Cullen DJ, Civetta JM, Briggs BA, Ferrara LC. Therapeutic intervention scoring system: a method for quantitative comparison of patient care. Crit Care Med. 1974;2(2):57-60.

6. Miranda DR, de Rijk A, Schaufeli W. Simplified Therapeutic Intervention Scoring System: the TISS-28 items--results from a multicenter study. Crit Care Med. 1996;24(1):64-73.

7. Miranda DR. Nine equivalents of nursing manpower use score (NEMS). Intensive Care Med. 1997;23(7):760-5.

8. Stafsetha SK, Solms D, Bredal IS. The characterisation of workloads and nursing staff allocation in intensive care units: descriptive study using the Nursing Activities Score for the first time in Norway. Intensive Crit Care Nurs. 2011;27(5):290-4.

9. Donabedian A. Models for organizing the delivery of personal health services and criteria for evluating them. Milbank Mem Fund Q. 1972;50(4):103-54.

10. Whittemore R, Knafl K. The integrative review: updated methodology. J Adv Nurs. 2005;52(5):546-53.

11. Moher D, Liberati A, Tetzlaff J, Altman DG, Group P. Preferred reporting items for systematic reviews and meta-analyses: the PRISMA statement. J Clin Epidemiol. 2009;62(10):1006-12.

12. Stelfox HT, Lane D, Boyd JM, Taylor S, Perrier L, Straus S, et al. A scoping review of patient discharge from intensive care: opportunities and tools to improve care. Chest. 2015;147(2):317-27. 
13. Ski CF, Munian S, Rolley JX, Thompson DR. Evaluation of nurses' ${ }^{\prime}$ perceptions of the impact of targeted depression education and a screening and referral tool in an acute cardiac setting. J Clin Nurs. 2015;24(1-2):235-43.

14. Adell AB, Campos RA, Rey MCB, Rochera ES, Munoz JS, Canuto MS, et al. Nursing Activity Score (NAS). Nuestra experiencia con un sistema de computo de cargas de enfermaria basado en tiempos. Enferm Intensiva. 2005;16(4):164-73.

15. Altafin JA, Grion CM, Tanita MT, Festti J, Cardoso LT, Veiga CF, et al. Nursing Activities Score and workload in the intensive care unit of a university hospital. Rev Bras Ter Intensiva. 2014;26(3):292-8.

16. Argibay-Lago A, Fernandez-Rodriguez D, Ferrer-Sala N, Prieto-Robles C, Hernanz-del Rio A, Castro-Rebollo P. Evaluation of nurse workload in patients undergoing therapeutic hypothermia. Enferm Clin. 2014;24(6):323-9.

17. Camuci MB, Martins JT, Cardeli AAM, Cruz Robazzi MLDC. Nursing Activities Score: nursing work load in a burns intensive care unit. Rev Latino Am Enfermagem. 2014;22(2):325-31.

18. Carmona-Monge FJ, Jara-Pérez A, Quiros-Herranz C, Rollan-Rodriguez G, Cerrillo-Gonzalez I, S. G-G, et al. Assessment of nursing workload in three groups of patients in a spanich ICU the nursing activities scores scale. Rev Esc Enferm USP. 2013;47(2):335-40.

19. Carmona-Monge FJ, Uranga IU, Gomez SG, Herranz CQ, Bengoetxea MB, Unanue GE, et al. Usage analysis of the Nursing Activities Score in two Spanish ICUS. Rev Esc Enferm USP. 2013;47(5):1108-16.

20. Ciampone JT, Goncalves LA, Maia FDOM, Padilha KG. Nursing care need and therapeutics interventions in intensive care unit: a comparative study among elderly and non-elderly. Acta Paul Enferm. 2006;19(1):28-35.

21. Coelho FUDA, Queijo AF, Andolhe R, Goncalves LA, Padilha KG. Carga de trabalho de enfermagem em unidade de terapia intensiva de cargiologia e fatores clinicos associados. Texto Contexto Enferm. 2011;20(4):735-41.

22. Cremasco MF, Wenzel F, Zanei SS, Whitaker IY. Pressure ulcers in the intensive care unit: the relationship between nursing workload, illness severity and pressure ulcer risk. J Clin Nurs. 2013;22(15-16):2183-91.

23. Cyrino CMS. Sitos assistenciais em unidade de terapia intensiva e relaçao do nursing activities score com a infecçao hospitalar. Esc Anna Nery. 2012;16(4):712-8.

24. Silva MCM, Sousa RMC, Padilha KG. Patient destination after discharge from intensive care units: wards or intermediate care units. Rev Latino Am Enfermagem. 2012;18(2):224-32.

25. Silva MCM, Sousa RMC, Padilha KG. Factors associated with death and readmission into the intensive care unit. Rev Latino Am Enfermagem. 2011;19(4):911-9.

26. Daud-Gallotti RM, Costa SF, Guimaraes T, Padilha KG, Inoue EN, Vasconcelos TN, et al. Nursing workload as a risk factor for healthcare associated infections in ICU: a prospective study. PLoS One. 2012;7(12):e52342.

27. Sousa CR, Goncalves LA, Toffoleto MC, Leao K, Padilha KG. Predictors of nursing workload in elderly patients admitted to intensive care units. Rev Latino Am Enfermagem. 2008;16(2):218-23.

28. Feitosa MC, Leite IRL, Silva GRF. Demanda de intervençoes de enfermagem a pacientes sob cuidados intensivos: NAS - Nursing Activities Score. Esc Anna Nery. 2012;16(4):682-8.

29. Giakoumidakis K, Baltopoulos Gl, Charitos C, Patelarou E, Galanis P, Brokalaki H. Risk factors for prolonged stay in cardiac surgery intensive care units. Nurs Crit Care. 2011;16(5):243-51.

30. Gerasimou-Angelidi S, Myrianthefs P, Chovas A, Baltopoulos G, Komnos A. Nursing Activities Score as a predictor of family satisfaction in an adult intensive care unit in Greece. J Nurs Manag. 2014;22(2):151-8.

31. Giakoumidakis K, Baltopoulos GI, Charitos C, Patelarou E, Fotos NV, Brokalaki-Pananoudaki H. Risk factors for increased in-hospital mortality: a cohort study among cardiac surgery patients. Eur J Cardiovasc Nurs. 2012;11(1):23-33.

32. Goncalves LA, Garcia PC, Toffoleto MC, Telles SCR, Padilha KG. Necessidades de cuidadis de enfermagem em Terapia Intensiva: evoluçao diaria dos patientes segundo o Nursing Activites Score (NAS). Rev Bras Enferm. 2006;59(1):56-60.

33. Goncalves LA, Padilha KG. Fatores associados à carga de trabalho de enfermagem em Unidade de Terapia Intensiva. Rev Esc Enferm USP. 2007;41(4):645-52.

34. Inoue KC, Matsuda LM. Sizing the nursing staff in an intensive care unit for adults. Acta Paul Enferm. 2010;23(3):379-84.

35. Kakushi LE, Evora YD. Direct and indirect nursing care time in an intensive care unit. Rev Latino Am Enfermagem. 2014;22(1):150-7.

36. Lago PM, Nilson C, Piva JP, Vieira AC, Halal MG, Abib GMDC, et al. Nurses' participation in the end-of-life process in two paediatric intensive care units in Brazil. International. J Palliat Nurs. 2011;17(6):264-70.

37. Lucchini A, Peruta M, Canella R, Elli S, Sanvitos G, De Angelis C, et al. Fabbisogno assistenziale e insorgenza di eventi avversi in terapia intensiva: i risultati di un'indagine. Assist Inferm Ric. 2011;30:172-9.

38. Lucchini A, Elli S, Bambi S, Foti G, Fumagalli R. Ventilazione invasiva e non invasiva: impatto sul carico di lavoro infermieristico. Assist Inferm Ric. 2012;32:124-31.

39. Lucchini A, De Felippis C, Elli S, Schifano L, Rolla F, Pegoraro F, et al. Nursing Activities Score (NAS): 5 years of experience in the intensive care units of an Italian University hospital. Intensive Crit Care Nurs. 2014;30(3):152-8.

40. Nogueira LDS, Santos MR, E. MS, Moock M. Nursing Activities Score: comparison among the index APACHE II and the mortality in patients admitted in intensive care unit. Rev Bras Ter Intensiva. 2007;19(3):327-30.

41. Nogueira LDS, Koike KM, Sardinha DS, Padilha KG, Sousa RMC. Nursing workload in public and private intensive care units. Rev Bras Ter Intensiva. 2013;25(3):225-32.

42. Nogueira LDS, Domingues CDA, Poggetti RS, Sousa RMC. Nursing workload in intensive care unit trauma patients: analysis of associated factors. PLoS One. 2014;9(11):e112125. 
43. Novaretti MCZ, Santos EDV, Quitério LM, Daud-Gallotti RM. Sobrecarga de trabalho da enfermagem e incidentes e eventos adversos em pacientes internados em UTI. Rev Bras Enferm. 2014;67(5):692-9.

44. Nunes BK, Toma E. Assessment of a neonatal unit nursing staff: application of the Nursing Activities Score. Rev Latino Am Enfermagem. 2013;21(1):348-55.

45. Padilha KG, de Sousa RMC, Queijo AF, Mendes AM, Miranda DR. Nursing Activities Score in the intensive care unit: Analysis of the related factors. Intensive Crit Care Nurs. 2008;24(3):197-204

46. Padilha KG, Sousa RM, Garcia PC, Bento ST, Finardi EM, Hatarashi RH. Nursing workload and staff allocation in an intensive care unit: a pilot study according to Nursing Activities Score (NAS). Intensive Crit Care Nurs. 2010;26(2):108-13.

47. Panunto MR, Guirardello EDB. Nursing wokload in an intensive care unit of a teaching hospital. Acta Paul Enferm. 2012;25(1):96-101.

48. Peng L, Mayner L, Wang H. Association between trauma patients' severity and critical care nursing workload in China. Nurs Health Sci. 2014;16(4):528-33.

49. Queijo AF, Martins RS, Andolhe R, Oliveira EM, Barbosa RL, Padilha KG. Nursing workload in neurological intensive care units: cross-sectional study. Intensive Crit Care Nurs. 2013;29(2):112-6.

50. McDonald KM, Sundaram V, Bravata DM. Conceptual frameworks and their application to evaluating care coordination interventions. In: Closing the Qulity Gap: critical analysis of qulity improvement strategies. Rockville: Agency for Healthcare Research and Quality; 2007. (Technical Reviews, n. 97).

51. Young MP, Birkmeyer JD. Potential reduction in mortality rates using an intensivist model to manage intensive care units. Eff Clin Pract. 2000;3(6):284-9.

Conflict of interest: the authors declare that there is no conflict of interests regarding the publication of this paper.

Financial support: this study was funded through a subvention provided by Réseau de Recherche en Interventions en Sciences Infirmières du Québec (RRISIQ). 